FACTA UNIVERSITATIS (NIŠ)

Ser. Math. Inform. Vol. 35, No 4 (2020), 1107-1125

https://doi.org/10.22190/FUMI2004107B

\title{
STATISTICAL INFERENCE FOR GEOMETRIC PROCESS WITH THE GENERALIZED RAYLEIGH DISTRIBUTION
}

\author{
Cenker Biçer, Hayrinisa D. Biçer, Mahmut Kara and Asuman Yılmaz
}

(C) 2020 by University of Niš, Serbia | Creative Commons Licence: CC BY-NC-ND

\begin{abstract}
In the present paper, the statistical inference problem is considered for the geometric process (GP) by assuming the distribution of the first arrival time with generalized Rayleigh distribution with the parameters $\alpha$ and $\lambda$. We have used the maximum likelihood method for obtaining the ratio parameter of the GP and distributional parameters of the generalized Rayleigh distribution. By a series of Monte-Carlo simulations evaluated through the different samples of sizes - small, moderate and large, we have also compared the estimation performances of the maximum likelihood estimators with the other estimators available in the literature such as modified moment, modified L-moment, and modified least squares. Furthermore, wehave presented two real-life datasets analyses to show the modeling behavior of GP with generalized Rayleigh distribution.
\end{abstract}

Keywords: Monotone processes; non-parametric estimation; parametric estimation; stochastic process; data with trend.

\section{Introduction}

In 1988, Lam [18] introduced the geometric process (GP) as a simple monotonic stochastic process. In order to model a successive inter-arrival times dataset with a monotone trend, the GP is a quite important alternative to the alpha series process and the nonhomogeneous Poisson process with a monotone intensity function. Since it has a simple form which is easily applied to the many real-life problems from different areas such as science, health, engineering etc., see [17], its popularity increases day by day according to its alternatives. Some key features of the GP and its advantages, which the GP provides in the modeling of the arrival times data with a trend, studied by Lam [16], Lam [18], Lam et al.[19] and Braun et al. [9], [10]. The GP is given by the following definition, see [17].

Received August 29, 2019; accepted April 12, 2020

2020 Mathematics Subject Classification. Primary 60G55; Secondary 60K05, 62F12 


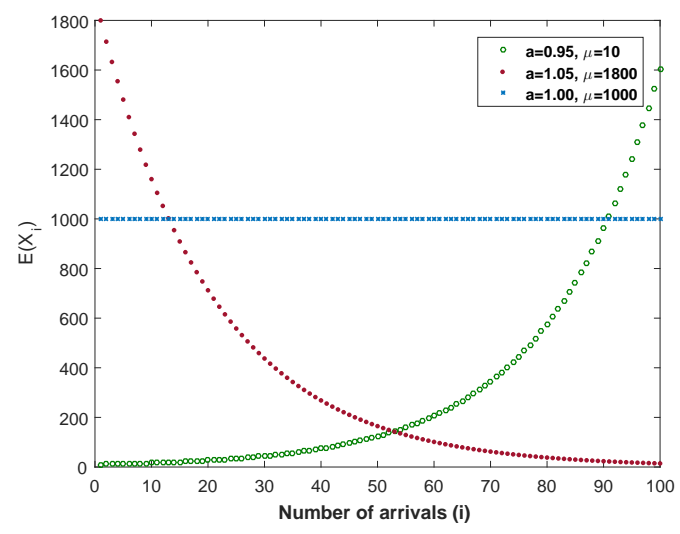

FIG. 1.1: Behavior of the GP

Definition 1.1. Let $X_{i}$ be the arrival time between the $(i-1)$ th and $i$ th events of a counting process $\{N(t), t \geq 0\}$ for $i=1,2, \ldots$. The process $\left\{X_{i}, i=1, \ldots, n\right\}$ is said to be a GP with parameter $a$ if there exists a real number $a>0$ such that $Y_{i}=a^{i-1} X_{i}, i=1,2, \ldots$, are independently and identically distributed (iid) random variables which have any continuous distribution supported on positive real interval. Where $a$ is called the ratio parameter of the GP.

In a general concept, there are three important parameter types in a GP. The first of these parameter types is the ratio parameter $a$. The second type of them is mean and variance of the first arrival time $X_{1}$. In the GP, determining the mean and variance of the first arrival time is quite important because of the fact that the means and variances of the random variables $X_{i}, i=1,2, \ldots$ are easily represented by the mean and variance of the first arrival time. Assume that $E\left(X_{1}\right)=\mu$ and $\operatorname{Var}\left(X_{1}\right)=\sigma^{2}$ for a GP with the ratio parameter $a$. By these notations, the mean and variance of the random variable $X_{i},(i=1,2, \cdots, n)$, are given by following forms:

$$
\begin{gathered}
E\left(X_{i}\right)=\frac{\mu}{a^{i-1}}, i=1,2, \ldots \\
\operatorname{Var}\left(X_{i}\right)=\frac{\sigma^{2}}{a^{2(i-1)}}, i=1,2, \ldots
\end{gathered}
$$

Hence, by using the relation given by equation 1.1, we can provide Figure 1.1 to illustrate the monotonic behavior of the GP, where the $E\left(X_{i}\right)$ is plotted against the arrival number $i,(i=1,2, \cdots$,$) for a fixed \mu$.

By the Figure 1.1, the process has a monotone increasing behavior when $a<1$ and has a monotone decreasing behavior when $a>1$. If $a=1$ then the process is a Renewal process (RP) [17]. 
The last type of the important parameters is the distributional parameters of the first occurrence time $X_{1}$. In the literature, one can find many published studies related to the parameter estimation problem for both the ratio parameter $a$ and distributional parameters of GP. Lam [16] obtained some non-parametric estimators for parameter $a$. Several studies that take into account some specific lifetime distributions for first occurrence time $X_{1}$ and focus on estimating the distributional parameters of GP are as follows: Gamma [12], Weibull [3], log-normal [18], inverse Gaussian [13], Lindley [7], power Lindley [4], Rayleigh [8], two-parameter Rayleigh [5] and two-parameter Lindley [6] distribution for the GP.

The main motivation of this study is to estimate the parameters of GP when the distribution of first occurrence time is Generalized Rayleigh (GR) also known as two-parameter Burr Type X distribution. We are motivated to the GR distribution for the distribution of the first occurrence time because it is an important alternative to the other famous distributions used in reliability analysis such as the Gamma, Weibull, exponential. In accordance with the purpose of this study, we employ the maximum likelihood (ML), modified moments (MM), modified L-moments (MLM) and modified least-squares (MLS) methods to obtain estimators of the unknown parameters of GP.

The rest of the paper is organized as follows: In section 2, we shall overview the GR distribution. In section 3, we shall obtain the ML estimators of the unknown parameters of GP with the GR distribution. Furthermore, we will investigate some modified estimators for distributional parameters of GP considering the non-parametric estimate of the ratio parameter $a$. In section 4, some Monte-Carlo simulation studies which compare the efficiencies of the ML estimators obtained in section 3 with the MM, the MLM, and the MLS estimators are performed. Section 5 covers two real-life examples which illustrate the modeling capability of a GP with GR distribution. Section 6 concludes the study.

\section{An overview to GR distribution}

The GR distribution, also known as two-parameter Burr Type $\mathrm{X}$ distribution, was originally studied by Surles and Padgett [22]. Later on, the distribution was renamed as the GR by Raqab and Kundu [21]. The GR is a commonly used probability model in the modeling of positive and non-symmetric data observed from various areas such as communication, health, engineering, reliability etc. Since the distribution is applicable to the modeling of data measured from a wide variety of areas, the interest in the theory and methods related to GR distribution is progressive.

The probability density function (pdf) of the GR distribution with the parameters $\alpha$ and $\lambda$ is

$$
f(x ; \alpha, \lambda)=2 \alpha \lambda^{2} x e^{-(\lambda x)^{2}}\left(1-e^{-(\lambda x)^{2}}\right)^{\alpha-1}, x>0,
$$




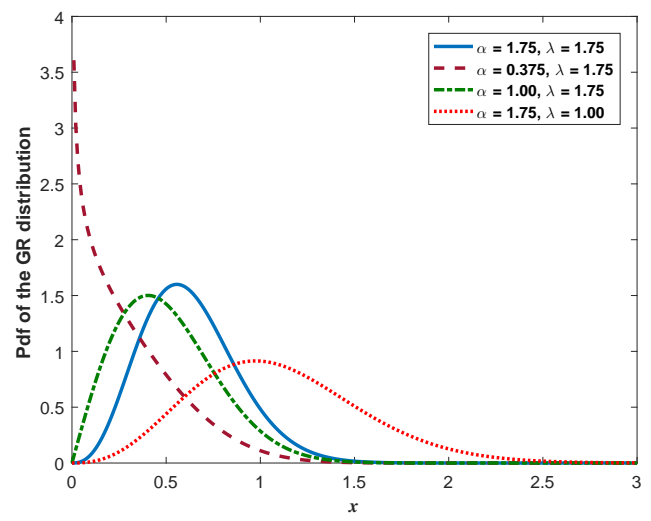

FIG. 2.1: Pdf of the GR distribution for the different values of the parameters

and the corresponding cumulative distribution (cdf) is

$$
F(x, \alpha, \lambda)=\left(1-e^{-(\lambda x)^{2}}\right)^{\alpha}, x>0,
$$

where $\alpha$ and $\lambda$ are the positive and real valued scale and shape parameters of the distribution, respectively [14]. When $\alpha=1$, the GR distribution is a Rayleigh with parameter $\lambda$. If $\lambda=1$, then the distribution is reduce to the one-parameter Burr Type $\mathrm{X}$ distribution with parameter $\alpha$. The GR distribution is a unimodal and its pdf is skew to the right when $\alpha>\frac{1}{2}$ and is a decreasing function otherwise [21]. Figure 2.1 below lucidly show the behaviors of the pdf of the GR distribution discussed in here.

The expectation and variance of the GR distribution are not available in the explicit forms, however, they can be easily obtained for selected values of the parameters by using a numeric method.

\section{Inference for GP}

In this section, in addition to obtaining the ML estimators of the GP with GR distribution, we will also investigate some modified estimators when the ratio parameter of the process is estimated by using a non-parametric estimator.

\subsection{Estimates}

Let us $X_{1}, X_{2}, \ldots, X_{n}$ be a random sample taken from a GP with ratio $a$ and $X_{1} \sim G R(\alpha, \lambda)$ with the pdf (2.1). By considering the equation (2.1) and Definition 1.1 , the log-likelihood function for the random variables $X_{i},(i=1,2, \ldots, n)$ can be written as 
$\ln L(a, \alpha, \lambda)=n(n-1) \ln a+n \ln 2+2 n \ln \lambda+n \ln \alpha-\lambda^{2} \sum_{i=1}^{n}\left(a^{i-1} x_{i}\right)^{2}$

$$
+\sum_{i=1}^{n} \ln x_{i}+(\alpha-1) \sum_{i=1}^{n} \ln \left(1-e^{-\left(\lambda a^{i-1} x_{i}\right)^{2}}\right) .
$$

If the first derivatives of Equation (3.1) according to $a, \alpha$ and $\lambda$ are taken, we have

$(3.2) \frac{\partial \ln L(a, \alpha, \lambda)}{\partial a}=\frac{(n-1) n}{a}+2(\alpha-1) \sum_{i=1}^{n} \frac{(i-1) \lambda^{2} a^{2 i-3} x_{i}^{2} e^{\lambda^{2}\left(-a^{2 i-2}\right) x_{i}^{2}}}{1-e^{\lambda^{2}\left(-a^{2 i-2}\right) x_{i}^{2}}}=0$

$$
\begin{gathered}
\frac{\partial \ln L(a, \alpha, \lambda)}{\partial \lambda}=\frac{2}{\lambda}+(\alpha-1) \sum_{i=1}^{n} \frac{2 \lambda a^{2 i-2} x_{i}^{2} e^{\lambda^{2}\left(-a^{2 i-2}\right) x_{i}^{2}}}{1-e^{\lambda^{2}\left(-a^{2 i-2}\right) x_{i}^{2}}}=0 \\
\frac{\partial \ln L(a, \alpha, \lambda)}{\partial \alpha}=\frac{n}{\alpha}+\sum_{i=1}^{n} \log \left(1-e^{\lambda^{2}\left(-a^{2 i-2}\right) x_{i}^{2}}\right)
\end{gathered}
$$

analytical expressions for the ML estimators of the parameters $a, \lambda$ and $\alpha$ can not be obtained from equations (3.2)-(3.4). However, equations (3.2)-(3.4) can be simultaneously solved using a numerical method such as well-known Newton's method.

Let $\theta=\left[\begin{array}{c}a \\ \lambda \\ \alpha\end{array}\right]$ be the parameter vector and likelihood equations given by (3.2)(3.3) and (3.4) are represented by a gradient vector $\nabla(\theta)$ as

$$
\nabla(\theta)=\left[\begin{array}{c}
\frac{\partial \ln L(a, \alpha, \lambda)}{\partial a(a, \alpha, \lambda)} \\
\frac{\partial \ln L(a, \alpha, \lambda}{\partial \lambda} \\
\frac{\partial \ln L(a, \alpha, \lambda)}{\partial \alpha}
\end{array}\right]
$$

Thus, in order to estimate of the parameter vector $\theta$, the iterative method given by 3.6 can be used by starting from an initial estimation such as $\hat{\theta}_{0}$.

$$
\theta_{m+1}=\theta_{m}-H^{-1}\left(\theta_{m}\right) \nabla\left(\theta_{m}\right)
$$

where $H^{-1}(\theta)$ is the inverse of the Hessian matrix $H(\theta)$. The elements of the matrix $H(\theta)$ are the second derivatives of the log-likelihood function (3.1) with respect to $a, \lambda$ and $\alpha$. Let $h_{i j}$ be the $(i, j)$ th $(i, j=1,2,3)$ element of the matrix $H(\theta)$. The $h_{i j}$ 's are obtained as below

$$
\begin{aligned}
h_{11}= & -\frac{(n-1) n}{a^{2}}+(\alpha-1) \sum_{i=1}^{n}\left(\frac{(2 i-3)(2 i-2) \lambda^{2} a^{2 i-4} x_{i}^{2} e^{\lambda^{2}\left(-a^{2 i-2}\right) x_{i}^{2}}}{1-e^{\lambda^{2}\left(-a^{2 i-2}\right) x_{i}^{2}}}\right. \\
& \left.-\frac{(2 i-2)^{2} \lambda^{4} a^{4 i-6} x_{i}^{4} e^{\lambda^{2}\left(-a^{2 i-2}\right) x_{i}^{2}}}{1-e^{\lambda^{2}\left(-a^{2 i-2}\right) x_{i}^{2}}}-\frac{(2 i-2)^{2} \lambda^{4} a^{4 i-6} x_{i}^{4} e^{-2 \lambda^{2} a^{2 i-2} x_{i}^{2}}}{\left(1-e^{\lambda^{2}\left(-a^{2 i-2}\right) x_{i}^{2}}\right)^{2}}\right)
\end{aligned}
$$




$$
\begin{gathered}
h_{13}=\sum_{i=1}^{n} \frac{(2 i-2) \lambda^{2} a^{2 i-3} x_{i}^{2} e^{\lambda^{2}\left(-a^{2 i-2}\right) x_{i}^{2}}}{1-e^{\lambda^{2}\left(-a^{2 i-2}\right) x_{i}^{2}}} \\
h_{22}=\quad-\frac{2}{\lambda^{2}}+(\alpha-1) \sum_{i=1}^{n}\left(\frac{2 a^{2 i-2} x_{i}^{2} e^{\lambda^{2}\left(-a^{2 i-2}\right) x_{i}^{2}}}{1-e^{\lambda^{2}\left(-a^{2 i-2}\right) x_{i}^{2}}}-\right. \\
\left.\frac{4 \lambda^{2} a^{4 i-4} x_{i}^{4} e^{\lambda^{2}\left(-a^{2 i-2}\right) x_{i}^{2}}}{1-e^{\lambda^{2}\left(-a^{2 i-2}\right) x_{i}^{2}}}-\frac{4 \lambda^{2} a^{4 i-4} x_{i}^{4} e^{-2 \lambda^{2} a^{2 i-2} x_{i}^{2}}}{\left(1-e^{\lambda^{2}\left(-a^{2 i-2}\right) x_{i}^{2}}\right)^{2}}\right) \\
h_{23}=\sum_{i=1}^{n} \frac{2 \lambda a^{2 i-2} x_{i}^{2} e^{\lambda^{2}\left(-a^{2 i-2}\right) x_{i}^{2}}}{1-e^{\lambda^{2}\left(-a^{2 i-2}\right) x_{i}^{2}}} \\
h_{33}=-\frac{n}{\alpha^{2}} .
\end{gathered}
$$

Note that inverse of the matrix $H$ is calculated as

$$
H^{-1}=\frac{1}{\operatorname{Det}(H)}\left[\begin{array}{ccc}
h_{22} h_{33}-h_{23} h_{32} & -h_{12} h_{33}-h_{13} h_{32} & h_{12} h_{23}-h_{13} h_{22} \\
-h_{21} h_{33}-h_{31} h_{23} & h_{11} h_{33}-h_{13} h_{31} & -h_{11} h_{23}-h_{21} h_{13} \\
h_{21} h_{32}-h_{22} h_{31} & -h_{11} h_{32}-h_{12} h_{31} & h_{11} h_{22}-h_{12} h_{21}
\end{array}\right],
$$

where $\operatorname{Det}(H)=h_{11} h_{22} h_{33}-h_{11} h_{23} h_{32}-h_{12} h_{21} h_{33}+h_{12} h_{31} h_{23}+h_{21} h_{13} h_{32}-$ $h_{13} h_{22} h_{31}$ is determinant of the matrix $H$. In the Newton method, iterations continue until $\left\|\theta_{m+1}-\theta_{m}\right\|<\varepsilon$ where $\varepsilon$ is a predetermined small constant and $\|\cdot\|$ is the Euclidean norm of a vector. Thus, ML estimators of the parameters of GP with GR distribution, say $\hat{a}_{M L}, \hat{\alpha}_{M L}$ and $\hat{\lambda}_{M L}$, are obtained from respective elements of $\theta_{m+1}$.

Now we investigate the asymptotic features of the estimators $\hat{a}_{M L}, \hat{\alpha}_{M L}$ and $\hat{\lambda}_{M L}$. The joint distribution of $\hat{a}_{M L}, \hat{\alpha}_{M L}$ and $\hat{\lambda}_{M L}$ is asymptotic-Normal $(A N)$ with mean vector $(a, \lambda, \alpha)$ and covariance $I^{-1}$, where matrix $I$ refers to Fisher information defined as

$$
I=-\frac{1}{n}\left[\begin{array}{lll}
E\left(\frac{\partial \ln L(a, \lambda, \alpha)}{\partial a^{2}}\right) & E\left(\frac{\partial \ln L(a, \lambda, \alpha)}{\partial a \partial \lambda}\right) & E\left(\frac{\partial \ln L(a, \lambda, \alpha)}{\partial a \partial \alpha}\right) \\
E\left(\frac{\partial \ln L(a, \lambda, \alpha)}{\partial a \partial \lambda}\right) & E\left(\frac{\partial \ln L(a, \lambda, \alpha)}{\partial \lambda^{2}}\right) & E\left(\frac{\partial \ln L(a, \lambda, \alpha)}{\partial \lambda \partial \alpha}\right) \\
E\left(\frac{\partial \ln L(a, \lambda, \alpha)}{\partial a \partial \alpha}\right) & E\left(\frac{\partial \ln L(a, \lambda, \alpha)}{\partial \lambda \partial \alpha}\right) & E\left(\frac{\partial \ln L(a, \lambda, \alpha)}{\partial \alpha^{2}}\right)
\end{array}\right] .
$$

The elements of the matrix $I$ are written from elements of the Hessian matrix. 


\subsection{Modified Methods}

Lam [16] introduced a non-parametric estimator to estimate only the ratio parameter of the process without making a specific distribution assumption for the GP. The non-parametric estimator of the ratio parameter $a$ is given by, see [16],

$$
\hat{a}_{N P}=\exp \left(\frac{6}{(n-1) n(n+1)} \sum_{i=1}^{n}(n-2 i+1) \ln X_{i}\right) .
$$

The distributional parameters of the GP are easily estimated using the available estimators in the literature when the ratio parameter $a$ is estimated as $\hat{a}_{N P}$. This approximation is known as modified estimation technique in the literature. Now we examine the estimates of the distributional parameters of GP with the GR distribution by assuming that the parameter $a$ is estimated as $\hat{a}_{N P}$. Let $X_{1}, X_{2}, \ldots, X_{n}$ be a random sample from a GP with ratio $a$ and $X_{1} \sim G R(\alpha, \lambda)$, and the parameter $a$ is known as $\hat{a}_{N P}$, from Definition 1.1, we have

$$
\hat{Y}_{i}=\hat{a}_{N P}^{i-1} X_{i}
$$

and $\hat{Y}_{i} \sim G R(\alpha, \lambda)$. Thus, the MM, MLM, and MLS estimators of the $\alpha$ and $\lambda$ parameters can be obtained as follows by taking into account the moments, Lmoments, and least-squares estimators given in [14] and along with the predicted $\hat{Y}_{i}$.

$M M$ Estimators: The MM estimate of the parameters $\alpha$, say $\hat{\alpha}_{M M}$ can be obtained from numerical solution of the equation

$$
\frac{\psi^{\prime}(1)-\psi^{\prime}(\alpha+1)}{(\psi(\alpha+1)-\psi(1))^{2}}-\frac{V}{U^{2}}=0
$$

where $U=\frac{1}{n} \sum_{i=1}^{n} \hat{Y}_{i}^{2}, V=\frac{1}{n} \sum_{i=1}^{n} \hat{Y}_{i}^{4}-U^{2}$ and $\psi($.$) is the digamma function,$ (cf. [1]). Also, by considering $\hat{\alpha}_{M M}$, MM estimates of the parameter $\lambda$, say $\hat{\lambda}_{M M}$ is obtained as follows

$$
\hat{\lambda}_{M M}=\sqrt{\frac{\psi\left(\hat{\alpha}_{M M}+1\right)-\psi(1)}{U}}
$$

MLM Estimators: The MLM estimates of the parameters $\alpha$ and $\lambda$, say $\hat{\alpha}_{M L M}$ and $\hat{\lambda}_{M L M}$, respectively, are obtained by numerical solution of non-linear equation

$$
\frac{\psi(2 \alpha+1)-\psi(\alpha 1)}{\psi(\alpha+1)-\psi(1)}-\frac{l_{2}}{l_{1}}=0,
$$

where $l_{1}=\frac{1}{n} \sum_{i=1}^{n} \hat{Y}_{(i)}^{2}$ and $l_{2}=\frac{2}{n(n-1)} \sum_{i=1}^{n}(i-1) \hat{Y}_{(i)}^{4}-l_{1}$ and notation $\hat{Y}_{(i)}$ indicates the $i$ th observation of ordered sample, where $i=1,2, \ldots n$.

MLS Estimators: The MLS estimates of the parameters $\alpha$ and $\lambda, \hat{\alpha}_{M L S}$ and $\hat{\lambda}_{M L s}$, respectively, are obtained by minimizing the quadratic function $Q(\alpha, \lambda)$ 


$$
Q(\alpha, \lambda)=\sum_{i=1}^{n}\left(\left(1-e^{-\left(\lambda \hat{Y}_{(i)}\right)^{2}}\right)^{\alpha}-\frac{i}{n+1}\right)^{2}
$$

with respect to $\alpha$ and $\lambda$.

For details on deriving these estimators, we refer to [14].

\section{Monte-Carlo Simulation Study}

In this section, we run some Monte-Carlo simulations to show the estimation performance of ML and modified estimators obtained in the previous section. The main

goal of these Monte-Carlo studies, besides displaying the estimation performance of the ML estimators, compare its efficiency with the other estimators. Throughout the Monte-Carlo studies, we set the parameter values as $\lambda=1, \alpha=0.5,1$ and 2, and $a=0.90,0.95,1.05,1.10$. By the 1000 times replicated simulations conducted on the different samples of sizes $n=30,50,100$, we compute the means, biases and $n \times$ mean squared errors $(n \times$ MSE) for the ML, MM, MLM and MLS estimates for each collection of parameters. The simulated results are presented in Tables 1-3.

According to the simulation results in Tables 4.1-4.3, we can clearly say that the performances of all estimators are quite satisfactory in all cases. Besides, as the sample size $n$ increases, bias and $n \times$ MSE values of all estimators decrease. Thus, we can say that all estimators are asymptotically unbiassed and consistent. In addition, ML estimators outperform the other estimators in small, moderate and large sample sizes. 
Statistical Inference for Geometric Process with Generalized Rayleigh Distribution 1115

Table 4.1: The simulated Means, Biases and nxMSEs for the ML, MLS, MM and MLM estimators of the parameters $a, \alpha$ and $\lambda$, when $\alpha=0.5$ and $\lambda=1$.

\begin{tabular}{|c|c|c|c|c|c|c|c|c|c|c|c|}
\hline & & & & $\hat{a}$ & & & $\hat{\alpha}$ & & & $\hat{\lambda}$ & \\
\hline$a$ & $n$ & Method & Mean & Bias & $n \times \mathrm{MSE}$ & Mean & Bias & $n \times \mathrm{MSE}$ & Mean & Bias & $n \times \mathrm{MSE}$ \\
\hline \multirow[t]{12}{*}{0.90} & \multirow[t]{4}{*}{30} & ML & 0.9021 & 0.0021 & 0.0419 & 0.5526 & 0.0526 & 5.5639 & 1.0555 & 0.0555 & 20.2610 \\
\hline & & MLS & 0.9014 & 0.0014 & 0.1107 & 0.5099 & 0.0099 & 4.8498 & 1.0573 & 0.0573 & 52.0585 \\
\hline & & MM & 0.9014 & 0.0014 & 0.1107 & 0.5675 & 0.0675 & 16.1491 & 1.0724 & 0.0724 & 42.9262 \\
\hline & & MLM & 0.9014 & 0.0014 & 0.1107 & 0.4845 & 0.0155 & 6.6621 & 1.0213 & 0.0213 & 36.3018 \\
\hline & \multirow[t]{4}{*}{50} & ML & 0.8999 & 0.0001 & 0.0123 & 0.5280 & 0.0280 & 2.4407 & 1.0667 & 0.0667 & 16.1984 \\
\hline & & MLS & 0.9000 & 0.0000 & 0.0255 & 0.5073 & 0.0073 & 2.9507 & 1.0523 & 0.0523 & 26.8384 \\
\hline & & MM & 0.9000 & 0.0000 & 0.0255 & 0.5301 & 0.0301 & 8.9010 & 1.0528 & 0.0528 & 27.8249 \\
\hline & & MLM & 0.9000 & 0.0000 & 0.0255 & 0.4878 & 0.0122 & 3.8533 & 1.0313 & 0.0313 & 24.6382 \\
\hline & \multirow[t]{4}{*}{100} & ML & 0.8998 & 0.0002 & 0.0012 & 0.5172 & 0.0172 & 1.1802 & 1.0423 & 0.0423 & 6.5389 \\
\hline & & MLS & 0.8996 & 0.0004 & 0.0034 & 0.5057 & 0.0057 & 1.4203 & 1.0512 & 0.0512 & 15.3660 \\
\hline & & MM & 0.8996 & 0.0004 & 0.0034 & 0.5238 & 0.0238 & 3.9173 & 1.0601 & 0.0601 & 15.8429 \\
\hline & & MLM & 0.8996 & 0.0004 & 0.0034 & 0.4981 & 0.0019 & 1.5968 & 1.0451 & 0.0451 & 13.9685 \\
\hline \multirow[t]{12}{*}{0.95} & \multirow[t]{4}{*}{30} & ML & 0.9507 & 0.0007 & 0.0571 & 0.5590 & 0.0590 & 5.5451 & 1.1014 & 0.1014 & 29.9822 \\
\hline & & MLS & 0.9519 & 0.0019 & 0.1450 & 0.5160 & 0.0160 & 6.0655 & 1.0577 & 0.0577 & 52.0999 \\
\hline & & MM & 0.9519 & 0.0019 & 0.1450 & 0.5943 & 0.0943 & 16.8724 & 1.1040 & 0.1040 & 51.0806 \\
\hline & & MLM & 0.9519 & 0.0019 & 0.1450 & 0.4981 & 0.0019 & 6.6786 & 1.0463 & 0.0463 & 44.1838 \\
\hline & \multirow[t]{4}{*}{50} & ML & 0.9504 & 0.0004 & 0.0107 & 0.5335 & 0.0335 & 3.1944 & 1.0481 & 0.0481 & 14.6898 \\
\hline & & MLS & 0.9501 & 0.0001 & 0.0270 & 0.5089 & 0.0089 & 3.3424 & 1.0425 & 0.0425 & 27.6539 \\
\hline & & MM & 0.9501 & 0.0001 & 0.0270 & 0.5536 & 0.0536 & 9.9358 & 1.0675 & 0.0675 & 30.7541 \\
\hline & & MLM & 0.9501 & 0.0001 & 0.0270 & 0.4943 & 0.0057 & 4.3293 & 1.0319 & 0.0319 & 26.2168 \\
\hline & \multirow[t]{4}{*}{100} & ML & 0.9500 & 0.0000 & 0.0014 & 0.5207 & 0.0207 & 1.1473 & 1.0396 & 0.0396 & 6.5734 \\
\hline & & MLS & 0.9501 & 0.0001 & 0.0037 & 0.5094 & 0.0094 & 1.3092 & 1.0314 & 0.0314 & 13.4648 \\
\hline & & MM & 0.9501 & 0.0001 & 0.0037 & 0.5307 & 0.0307 & 3.9233 & 1.0442 & 0.0442 & 13.9762 \\
\hline & & MLM & 0.9501 & 0.0001 & 0.0037 & 0.5037 & 0.0037 & 1.4159 & 1.0291 & 0.0291 & 12.2366 \\
\hline \multirow[t]{12}{*}{1.05} & \multirow[t]{4}{*}{30} & ML & 1.0508 & 0.0008 & 0.0596 & 0.5529 & 0.0529 & 5.1782 & 1.0974 & 0.0974 & 24.8192 \\
\hline & & MLS & 1.0494 & 0.0006 & 0.1488 & 0.5149 & 0.0149 & 6.0798 & 1.0998 & 0.0998 & 57.9221 \\
\hline & & MM & 1.0494 & 0.0006 & 0.1488 & 0.5785 & 0.0785 & 16.3929 & 1.1198 & 0.1198 & 49.7872 \\
\hline & & MLM & 1.0494 & 0.0006 & 0.1488 & 0.4939 & 0.0061 & 6.8040 & 1.0689 & 0.0689 & 40.4968 \\
\hline & \multirow[t]{4}{*}{50} & ML & 1.0500 & 0.0000 & 0.0140 & 0.5296 & 0.0296 & 2.6421 & 1.0665 & 0.0665 & 13.1142 \\
\hline & & MLS & 1.0509 & 0.0009 & 0.0360 & 0.5079 & 0.0079 & 2.8651 & 1.0349 & 0.0349 & 28.8999 \\
\hline & & MM & 1.0509 & 0.0009 & 0.0360 & 0.5451 & 0.0451 & 8.0653 & 1.0553 & 0.0553 & 28.4833 \\
\hline & & MLM & 1.0509 & 0.0009 & 0.0360 & 0.4952 & 0.0048 & 3.2639 & 1.0263 & 0.0263 & 24.7169 \\
\hline & \multirow[t]{4}{*}{100} & ML & 1.0501 & 0.0001 & 0.0015 & 0.5150 & 0.0150 & 1.1322 & 1.0254 & 0.0254 & 6.3604 \\
\hline & & MLS & 1.0502 & 0.0002 & 0.0041 & 0.5037 & 0.0037 & 1.3424 & 1.0147 & 0.0147 & 11.7915 \\
\hline & & MM & 1.0502 & 0.0002 & 0.0041 & 0.5226 & 0.0226 & 3.5821 & 1.0238 & 0.0238 & 12.4582 \\
\hline & & MLM & 1.0502 & 0.0002 & 0.0041 & 0.4954 & 0.0046 & 1.4584 & 1.0079 & 0.0079 & 11.0757 \\
\hline \multirow[t]{12}{*}{1.10} & \multirow[t]{4}{*}{30} & ML & 1.1017 & 0.0017 & 0.0721 & 0.5657 & 0.0657 & 5.3227 & 1.0710 & 0.0710 & 21.8885 \\
\hline & & MLS & 1.1013 & 0.0013 & 0.1583 & 0.5205 & 0.0205 & 4.2594 & 1.0488 & 0.0488 & 41.9511 \\
\hline & & MM & 1.1013 & 0.0013 & 0.1583 & 0.5946 & 0.0946 & 16.2766 & 1.0973 & 0.0973 & 51.3694 \\
\hline & & MLM & 1.1013 & 0.0013 & 0.1583 & 0.5024 & 0.0024 & 5.8000 & 1.0408 & 0.0408 & 40.9797 \\
\hline & \multirow[t]{4}{*}{50} & ML & 1.1007 & 0.0007 & 0.0155 & 0.5321 & 0.0321 & 2.7774 & 1.0389 & 0.0389 & 12.7543 \\
\hline & & MLS & 1.1005 & 0.0005 & 0.0321 & 0.5114 & 0.0114 & 3.4238 & 1.0277 & 0.0277 & 23.1304 \\
\hline & & MM & 1.1005 & 0.0005 & 0.0321 & 0.5653 & 0.0653 & 10.4034 & 1.0609 & 0.0609 & 24.3047 \\
\hline & & MLM & 1.1005 & 0.0005 & 0.0321 & 0.5028 & 0.0028 & 4.2720 & 1.0227 & 0.0227 & 20.2996 \\
\hline & \multirow[t]{4}{*}{100} & ML & 1.1000 & 0.0000 & 0.0018 & 0.5140 & 0.0140 & 1.0566 & 1.0329 & 0.0329 & 5.8668 \\
\hline & & MLS & 1.1003 & 0.0003 & 0.0049 & 0.4964 & 0.0036 & 1.2135 & 1.0060 & 0.0060 & 12.3035 \\
\hline & & MM & 1.1003 & 0.0003 & 0.0049 & 0.5206 & 0.0206 & 3.7712 & 1.0252 & 0.0252 & 14.6663 \\
\hline & & MLM & 1.1003 & 0.0003 & 0.0049 & 0.4928 & 0.0072 & 1.4634 & 1.0076 & 0.0076 & 12.4369 \\
\hline
\end{tabular}


Table 4.2: The simulated Means, Biases and $n x M S E s$ for the ML, MLS, MM and MLM estimators of the parameters $a, \alpha$ and $\lambda$, when $\alpha=1$ and $\lambda=1$.

\begin{tabular}{|c|c|c|c|c|c|c|c|c|c|c|c|}
\hline & & & & $\hat{a}$ & & & $\hat{\alpha}$ & & & $\hat{\lambda}$ & \\
\hline$a$ & $n$ & Method & Mean & Bias & $n \times \mathrm{MSE}$ & Mean & Bias & $n \times \mathrm{MSE}$ & Mean & Bias & $n \times \mathrm{MSE}$ \\
\hline \multirow[t]{12}{*}{0.90} & \multirow[t]{4}{*}{30} & ML & 0.8995 & 0.0005 & 0.0226 & 1.1673 & 0.1673 & 34.3936 & 1.0710 & 0.0710 & 12.8072 \\
\hline & & MLS & 0.9002 & 0.0002 & 0.0364 & 1.0807 & 0.0807 & 37.1783 & 1.0255 & 0.0255 & 18.3058 \\
\hline & & MM & 0.9002 & 0.0002 & 0.0364 & 1.2559 & 0.2559 & 84.4448 & 1.0717 & 0.0717 & 18.4970 \\
\hline & & MLM & 0.9002 & 0.0002 & 0.0364 & 1.0727 & 0.0727 & 32.7471 & 1.0313 & 0.0313 & 15.3820 \\
\hline & \multirow[t]{4}{*}{50} & ML & 0.9002 & 0.0002 & 0.0050 & 1.0705 & 0.0705 & 12.2203 & 1.0226 & 0.0226 & 5.9189 \\
\hline & & MLS & 0.9006 & 0.0006 & 0.0081 & 1.0206 & 0.0206 & 15.2559 & 0.9911 & 0.0089 & 8.0817 \\
\hline & & MM & 0.9006 & 0.0006 & 0.0081 & 1.1228 & 0.1228 & 30.5716 & 1.0241 & 0.0241 & 9.9016 \\
\hline & & MLM & 0.9006 & 0.0006 & 0.0081 & 1.0159 & 0.0159 & 13.6941 & 0.9961 & 0.0039 & 7.9823 \\
\hline & \multirow[t]{4}{*}{100} & ML & 0.8999 & 0.0001 & 0.0006 & 1.0335 & 0.0335 & 5.5753 & 1.0233 & 0.0233 & 2.8179 \\
\hline & & MLS & 0.9000 & 0.0000 & 0.0009 & 1.0135 & 0.0135 & 7.6169 & 1.0095 & 0.0095 & 4.2659 \\
\hline & & MM & 0.9000 & 0.0000 & 0.0009 & 1.0668 & 0.0668 & 15.4622 & 1.0268 & 0.0268 & 4.8785 \\
\hline & & MLM & 0.9000 & 0.0000 & 0.0009 & 1.0127 & 0.0127 & 7.2168 & 1.0134 & 0.0134 & 3.9930 \\
\hline \multirow[t]{12}{*}{0.95} & \multirow[t]{4}{*}{30} & ML & 0.9486 & 0.0014 & 0.0302 & 1.1563 & 0.1563 & 32.0993 & 1.0953 & 0.0953 & 14.9887 \\
\hline & & MLS & 0.9482 & 0.0018 & 0.0468 & 1.0824 & 0.0824 & 46.1606 & 1.0610 & 0.0610 & 21.3359 \\
\hline & & MM & 0.9482 & 0.0018 & 0.0468 & 1.2546 & 0.2546 & 75.3895 & 1.1168 & 0.1168 & 21.4723 \\
\hline & & MLM & 0.9482 & 0.0018 & 0.0468 & 1.0828 & 0.0828 & 40.3227 & 1.0746 & 0.0746 & 18.0953 \\
\hline & \multirow[t]{4}{*}{50} & ML & 0.9500 & 0.0000 & 0.0061 & 1.0781 & 0.0781 & 16.7709 & 1.0411 & 0.0411 & 7.9158 \\
\hline & & MLS & 0.9499 & 0.0001 & 0.0101 & 1.0414 & 0.0414 & 25.0638 & 1.0266 & 0.0266 & 12.0983 \\
\hline & & MM & 0.9499 & 0.0001 & 0.0101 & 1.1250 & 0.1250 & 41.2553 & 1.0479 & 0.0479 & 11.8192 \\
\hline & & MLM & 0.9499 & 0.0001 & 0.0101 & 1.0263 & 0.0263 & 19.4910 & 1.0258 & 0.0258 & 10.3355 \\
\hline & \multirow[t]{4}{*}{100} & ML & 0.9500 & 0.0000 & 0.0007 & 1.0217 & 0.0217 & 4.4096 & 1.0115 & 0.0115 & 3.0601 \\
\hline & & MLS & 0.9501 & 0.0001 & 0.0012 & 1.0065 & 0.0065 & 6.4943 & 1.0016 & 0.0016 & 5.1477 \\
\hline & & MM & 0.9501 & 0.0001 & 0.0012 & 1.0593 & 0.0593 & 12.7412 & 1.0177 & 0.0177 & 4.8222 \\
\hline & & MLM & 0.9501 & 0.0001 & 0.0012 & 1.0033 & 0.0033 & 6.0221 & 1.0035 & 0.0035 & 4.3940 \\
\hline \multirow[t]{12}{*}{1.05} & \multirow[t]{4}{*}{30} & ML & 1.0494 & 0.0006 & 0.0265 & 1.1192 & 0.1192 & 21.9043 & 1.0618 & 0.0618 & 9.0571 \\
\hline & & MLS & 1.0495 & 0.0005 & 0.0429 & 1.0300 & 0.0300 & 19.9858 & 1.0261 & 0.0261 & 12.3809 \\
\hline & & MM & 1.0495 & 0.0005 & 0.0429 & 1.1917 & 0.1917 & 60.6033 & 1.0675 & 0.0675 & 14.1094 \\
\hline & & MLM & 1.0495 & 0.0005 & 0.0429 & 1.0257 & 0.0257 & 23.3553 & 1.0277 & 0.0277 & 11.3251 \\
\hline & \multirow[t]{4}{*}{50} & ML & 1.0500 & 0.0000 & 0.0075 & 1.0763 & 0.0763 & 10.4700 & 1.0345 & 0.0345 & 6.8339 \\
\hline & & MLS & 1.0497 & 0.0003 & 0.0105 & 1.0247 & 0.0247 & 13.3111 & 1.0186 & 0.0186 & 9.5556 \\
\hline & & MM & 1.0497 & 0.0003 & 0.0105 & 1.1251 & 0.1251 & 29.2849 & 1.0503 & 0.0503 & 10.9266 \\
\hline & & MLM & 1.0497 & 0.0003 & 0.0105 & 1.0229 & 0.0229 & 12.6927 & 1.0246 & 0.0246 & 9.1466 \\
\hline & \multirow[t]{4}{*}{100} & ML & 1.0502 & 0.0002 & 0.0007 & 1.0364 & 0.0364 & 4.7974 & 1.0122 & 0.0122 & 3.0109 \\
\hline & & MLS & 1.0502 & 0.0002 & 0.0013 & 1.0038 & 0.0038 & 5.7562 & 0.9983 & 0.0017 & 4.4178 \\
\hline & & MM & 1.0502 & 0.0002 & 0.0013 & 1.0683 & 0.0683 & 14.2285 & 1.0192 & 0.0192 & 5.3255 \\
\hline & & MLM & 1.0502 & 0.0002 & 0.0013 & 1.0061 & 0.0061 & 6.1276 & 1.0032 & 0.0032 & 4.4140 \\
\hline \multirow[t]{12}{*}{1.10} & \multirow[t]{4}{*}{30} & ML & 1.1003 & 0.0003 & 0.0353 & 1.1368 & 0.1368 & 27.3646 & 1.0665 & 0.0665 & 12.3611 \\
\hline & & MLS & 1.0998 & 0.0002 & 0.0460 & 1.0416 & 0.0416 & 30.0485 & 1.0269 & 0.0269 & 15.1012 \\
\hline & & MM & 1.0998 & 0.0002 & 0.0460 & 1.2349 & 0.2349 & 70.7584 & 1.0873 & 0.0873 & 17.8739 \\
\hline & & MLM & 1.0998 & 0.0002 & 0.0460 & 1.0502 & 0.0502 & 29.7390 & 1.0422 & 0.0422 & 14.3413 \\
\hline & \multirow[t]{4}{*}{50} & ML & 1.0999 & 0.0001 & 0.0075 & 1.1114 & 0.1114 & 15.9703 & 1.0530 & 0.0530 & 6.8821 \\
\hline & & MLS & 1.1001 & 0.0001 & 0.0113 & 1.0520 & 0.0520 & 17.7979 & 1.0204 & 0.0204 & 9.3748 \\
\hline & & MM & 1.1001 & 0.0001 & 0.0113 & 1.1819 & 0.1819 & 39.7175 & 1.0613 & 0.0613 & 10.0945 \\
\hline & & MLM & 1.1001 & 0.0001 & 0.0113 & 1.0592 & 0.0592 & 17.1944 & 1.0308 & 0.0308 & 8.3747 \\
\hline & \multirow[t]{4}{*}{100} & ML & 1.1001 & 0.0001 & 0.0010 & 1.0332 & 0.0332 & 4.9204 & 1.0139 & 0.0139 & 3.3641 \\
\hline & & MLS & 1.1000 & 0.0000 & 0.0017 & 1.0073 & 0.0073 & 6.3576 & 1.0052 & 0.0052 & 5.1185 \\
\hline & & MM & 1.1000 & 0.0000 & 0.0017 & 1.0602 & 0.0602 & 15.3053 & 1.0208 & 0.0208 & 5.3203 \\
\hline & & MLM & 1.1000 & 0.0000 & 0.0017 & 1.0062 & 0.0062 & 6.4213 & 1.0080 & 0.0080 & 4.5479 \\
\hline
\end{tabular}


Statistical Inference for Geometric Process with Generalized Rayleigh Distribution 1117

Table 4.3: The simulated Means, Biases and nxMSEs for the ML, MLS, MM and MLM estimators of the parameters $a, \alpha$ and $\lambda$, when $\alpha=2$ and $\lambda=1$.

\begin{tabular}{|c|c|c|c|c|c|c|c|c|c|c|c|}
\hline \multirow[b]{2}{*}{$a$} & \multirow[b]{2}{*}{$n$} & \multirow[b]{2}{*}{ Method } & \multicolumn{3}{|c|}{$\hat{a}$} & \multicolumn{3}{|c|}{$\hat{\alpha}$} & \multicolumn{3}{|c|}{$\hat{\lambda}$} \\
\hline & & & Mean & Bias & $n \times \mathrm{MSE}$ & Mean & Bias & $n \times \mathrm{MSE}$ & Mean & Bias & $n \times \mathrm{MSE}$ \\
\hline \multirow[t]{12}{*}{0.90} & \multirow[t]{4}{*}{30} & ML & 0.8999 & 0.0001 & 0.0134 & 2.3764 & 0.3764 & 185.0170 & 1.0691 & 0.0691 & 8.3586 \\
\hline & & MLS & 0.9001 & 0.0001 & 0.0172 & 2.2418 & 0.2418 & 399.8574 & 1.0313 & 0.0313 & 8.9383 \\
\hline & & MM & 0.9001 & 0.0001 & 0.0172 & 2.3288 & 0.3288 & 116.3478 & 1.0636 & 0.0636 & 9.1816 \\
\hline & & MLM & 0.9001 & 0.0001 & 0.0172 & 2.2279 & 0.2279 & 308.3195 & 1.0429 & 0.0429 & 8.3048 \\
\hline & \multirow[t]{4}{*}{50} & ML & 0.8996 & 0.0004 & 0.0031 & 2.1643 & 0.1643 & 70.3287 & 1.0346 & 0.0346 & 4.9931 \\
\hline & & MLS & 0.8997 & 0.0003 & 0.0037 & 2.0955 & 0.0955 & 105.0502 & 1.0178 & 0.0178 & 5.8255 \\
\hline & & MM & 0.8997 & 0.0003 & 0.0037 & 2.1857 & 0.1857 & 92.2488 & 1.0319 & 0.0319 & 5.8339 \\
\hline & & MLM & 0.8997 & 0.0003 & 0.0037 & 2.0743 & 0.0743 & 84.8229 & 1.0191 & 0.0191 & 5.3602 \\
\hline & \multirow[t]{4}{*}{100} & ML & 0.9000 & 0.0000 & 0.0003 & 2.0767 & 0.0767 & 22.3545 & 1.0111 & 0.0111 & 1.6830 \\
\hline & & MLS & 0.9000 & 0.0000 & 0.0004 & 2.0189 & 0.0189 & 29.2812 & 1.0009 & 0.0009 & 2.1476 \\
\hline & & MM & 0.9000 & 0.0000 & 0.0004 & 2.1333 & 0.1333 & 48.6809 & 1.0162 & 0.0162 & 2.3114 \\
\hline & & MLM & 0.9000 & 0.0000 & 0.0004 & 2.0183 & 0.0183 & 26.2329 & 1.0037 & 0.0037 & 1.8638 \\
\hline \multirow[t]{12}{*}{0.95} & \multirow[t]{4}{*}{30} & ML & 0.9503 & 0.0003 & 0.0124 & 2.3595 & 0.3595 & 168.6435 & 1.0486 & 0.0486 & 7.1311 \\
\hline & & MLS & 0.9502 & 0.0002 & 0.0153 & 2.1344 & 0.1344 & 159.8323 & 1.0111 & 0.0111 & 8.0305 \\
\hline & & MM & 0.9502 & 0.0002 & 0.0153 & 2.3240 & 0.3240 & 120.3577 & 1.0480 & 0.0480 & 7.8498 \\
\hline & & MLM & 0.9502 & 0.0002 & 0.0153 & 2.1655 & 0.1655 & 146.1292 & 1.0262 & 0.0262 & 7.5533 \\
\hline & \multirow[t]{4}{*}{50} & ML & 0.9498 & 0.0002 & 0.0030 & 2.1902 & 0.1902 & 74.3277 & 1.0317 & 0.0317 & 4.1722 \\
\hline & & MLS & 0.9498 & 0.0002 & 0.0035 & 2.0753 & 0.0753 & 84.4408 & 1.0100 & 0.0100 & 4.7112 \\
\hline & & MM & 0.9498 & 0.0002 & 0.0035 & 2.2398 & 0.2398 & 83.9141 & 1.0374 & 0.0374 & 4.8533 \\
\hline & & MLM & 0.9498 & 0.0002 & 0.0035 & 2.0776 & 0.0776 & 70.6279 & 1.0171 & 0.0171 & 4.3557 \\
\hline & \multirow[t]{4}{*}{100} & ML & 0.9501 & 0.0001 & 0.0004 & 2.1205 & 0.1205 & 38.9492 & 1.0084 & 0.0084 & 1.9344 \\
\hline & & MLS & 0.9501 & 0.0001 & 0.0005 & 2.0379 & 0.0379 & 40.9310 & 0.9959 & 0.0041 & 2.3678 \\
\hline & & MM & 0.9501 & 0.0001 & 0.0005 & 2.1333 & 0.1333 & 62.1434 & 1.0075 & 0.0075 & 2.6137 \\
\hline & & MLM & 0.9501 & 0.0001 & 0.0005 & 2.0401 & 0.0401 & 38.3668 & 0.9983 & 0.0017 & 2.2154 \\
\hline \multirow[t]{12}{*}{1.05} & \multirow[t]{4}{*}{30} & ML & 1.0503 & 0.0003 & 0.0185 & 2.2911 & 0.2911 & 164.7333 & 1.0347 & 0.0347 & 7.3818 \\
\hline & & MLS & 1.0505 & 0.0005 & 0.0218 & 2.0555 & 0.0555 & 211.5846 & 0.9842 & 0.0158 & 8.1717 \\
\hline & & MM & 1.0505 & 0.0005 & 0.0218 & 2.2707 & 0.2707 & 129.7693 & 1.0283 & 0.0283 & 8.5876 \\
\hline & & MLM & 1.0505 & 0.0005 & 0.0218 & 2.0674 & 0.0674 & 141.8400 & 1.0018 & 0.0018 & 7.7984 \\
\hline & \multirow[t]{4}{*}{50} & ML & 1.0499 & 0.0001 & 0.0032 & 2.1497 & 0.1497 & 67.0813 & 1.0352 & 0.0352 & 3.4191 \\
\hline & & MLS & 1.0500 & 0.0000 & 0.0039 & 2.0691 & 0.0691 & 97.2026 & 1.0112 & 0.0112 & 4.3589 \\
\hline & & MM & 1.0500 & 0.0000 & 0.0039 & 2.2045 & 0.2045 & 82.0182 & 1.0357 & 0.0357 & 4.0980 \\
\hline & & MLM & 1.0500 & 0.0000 & 0.0039 & 2.0698 & 0.0698 & 87.2862 & 1.0183 & 0.0183 & 3.7935 \\
\hline & \multirow[t]{4}{*}{100} & ML & 1.0500 & 0.0000 & 0.0005 & 2.0791 & 0.0791 & 26.5465 & 1.0111 & 0.0111 & 2.1213 \\
\hline & & MLS & 1.0500 & 0.0000 & 0.0007 & 2.0259 & 0.0259 & 39.8430 & 1.0009 & 0.0009 & 2.6829 \\
\hline & & MM & 1.0500 & 0.0000 & 0.0007 & 2.1326 & 0.1326 & 55.8938 & 1.0161 & 0.0161 & 2.8456 \\
\hline & & MLM & 1.0500 & 0.0000 & 0.0007 & 2.0264 & 0.0264 & 33.9811 & 1.0047 & 0.0047 & 2.4850 \\
\hline 1.10 & 30 & ML & 1.0998 & 0.0002 & 0.0174 & 2.3212 & 0.3212 & 194.9628 & 1.0429 & 0.0429 & 6.9826 \\
\hline & & MLS & 1.0997 & 0.0003 & 0.0214 & 2.1402 & 0.1402 & 165.2003 & 1.0096 & 0.0096 & 7.5882 \\
\hline & & MM & 1.0997 & 0.0003 & 0.0214 & 2.3034 & 0.3034 & 121.5048 & 1.0441 & 0.0441 & 8.2323 \\
\hline & & MLM & 1.0997 & 0.0003 & 0.0214 & 2.1614 & 0.1614 & 156.1144 & 1.0235 & 0.0235 & 7.5732 \\
\hline & 50 & ML & 1.1003 & 0.0003 & 0.0034 & 2.1643 & 0.1643 & 79.7157 & 1.0159 & 0.0159 & 2.9575 \\
\hline & & MLS & 1.1001 & 0.0001 & 0.0046 & 2.1007 & 0.1007 & 110.5767 & 1.0066 & 0.0066 & 4.3802 \\
\hline & & MM & 1.1001 & 0.0001 & 0.0046 & 2.1697 & 0.1697 & 97.4672 & 1.0176 & 0.0176 & 4.0407 \\
\hline & & MLM & 1.1001 & 0.0001 & 0.0046 & 2.0696 & 0.0696 & 90.7552 & 1.0067 & 0.0067 & 3.7026 \\
\hline & 100 & ML & 1.0997 & 0.0003 & 0.0006 & 2.1008 & 0.1008 & 30.4018 & 1.0329 & 0.0329 & 2.0447 \\
\hline & & MLS & 1.0997 & 0.0003 & 0.0007 & 2.0582 & 0.0582 & 40.5492 & 1.0243 & 0.0243 & 2.5109 \\
\hline & & MM & 1.0997 & 0.0003 & 0.0007 & 2.1426 & 0.1426 & 50.4873 & 1.0366 & 0.0366 & 2.8699 \\
\hline & & MLM & 1.0997 & 0.0003 & 0.0007 & 2.0540 & 0.0540 & 36.9373 & 1.0263 & 0.0263 & 2.4028 \\
\hline
\end{tabular}




\section{Application}

In this section, we analyze two real-life datasets called No.3 data and Software data to illustrate the estimation procedures the ML, the MM, the MLM and the MLS. To compare the RP and GPs with the ML, the MM, the MLM and the MLS estimators, we use the mean-squared error $\left(\mathrm{MSE}^{*}\right)$ criterion defined as, see [?],

$$
\text { - } \mathrm{MSE}^{*}=(1 / n) \sum_{k=1}^{n}\left(X_{k}-\hat{X}_{k}\right)^{2} \text {, }
$$

where $\hat{X}_{k}$ is calculated by

$$
\hat{X}_{k}=\left\{\begin{array}{cc}
\hat{\mu}_{(M L)} \hat{a}_{M L}^{1-k} & \text { GP with the ML estimators, } \\
\hat{\mu}_{(M L S)} \hat{a}_{N P}^{1-k} & \text { GP with the MLS estimators, } \\
\hat{\mu}_{(M M)} \hat{a}_{N P}^{1-k} & \text { GP with the MM estimators, } \\
\hat{\mu}_{(M L M)} \hat{a}_{N P}^{1-k} & \text { GP with the MLM estimators, } \\
\hat{\mu}_{(M L)} & \text { RP with the ML estimators, }
\end{array}\right.
$$

and $\hat{\mu}_{(.)}$is estimate of the expected value of the first occurrence time under the fitted $G R$ distribution with the ML, MM, MLM and MLS estimators and can be numerically calculated from

$$
\hat{\mu}_{(.)}=\int_{0}^{\infty} x f\left(x, \hat{\alpha}_{(.)}, \hat{\lambda}_{(.)}\right) d x
$$

No.3 data:

In the No.3 data set, there are 71 observations, which are regarding the unscheduled maintenance actions for U.S.S. Halfbeak No.3 main propulsion diesel engine [2]. This data set was found to be consistent with a GP in which the ratio parameter is greater than 1 , see [16].

In the first stage of data analysis, we investigate whether the data set follows a GR distribution. Linear regression model

$$
\ln X_{i}=\tau-(i-1) \ln a+\varepsilon_{i}
$$

can be employed to this aim, see [13] for further information on derivation of this regression model. Where $\tau=E\left(\ln Y_{i}\right), Y_{i}=a^{i-1} X_{i}$ and $\exp \left(\varepsilon_{i}\right) \sim G R(\theta, \beta)$. The error term $\varepsilon_{i}$ given in equation (5.2) can be easily estimated by

$$
\hat{\varepsilon}_{i}=\ln X_{i}-\hat{\tau}-(i-1) \ln \hat{a}_{N P}
$$

where $\hat{\tau}=\frac{n(n-1)}{2} \ln \hat{a}_{N P}+\sum_{i=1}^{n} \ln X_{i}$. Thus, we can say that the data set is consistent with a GR distribution if the exponential errors follow a GR distribution. The parameters estimations of the exponential errors are $\hat{\theta}_{M L}=0.2410$ and 

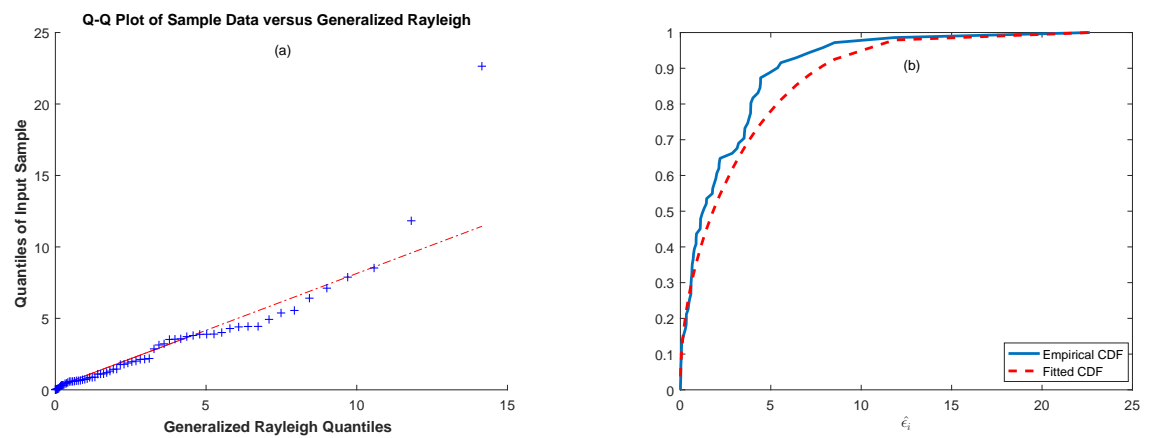

FIG. 5.1: QQ plot for the exponential errors (a), empirical and fitted cdf for the exponential errors (b)

Table 5.1: Estimation of parameters for the No 3 data set

\begin{tabular}{llcccc}
\hline Process & Method & $\hat{a}$ & $\hat{\alpha}$ & $\hat{\lambda}$ & $\mathrm{MSE} / 10^{5}$ \\
\hline GP & ML & 1.04272 & 0.12795 & 0.0002 & 1.93257 \\
\hline & MLS & & 0.2596 & 0.0004 & 2.0208 \\
& MM & \multirow{2}{*}{1.0416} & 0.0700 & 0.0002 & 2.2717 \\
& MLM & & 0.1277 & 0.0002 & 2.0210 \\
\hline RP & ML & 1.0000 & 0.1910 & 0.0007 & 3.3945 \\
\hline
\end{tabular}

$\hat{\beta}_{M L}=0.1330$ and also the value of Kolmogorov-Smirnov (K-S) test is 0.1286 and corresponding p-value is 0.1751 . Hence, result of the K-S test, we can say that the No. 3 dataset consistent with a GR distribution. To confirm this result, we present Figure 5.1(a) and Figure 5.1 (b). Figure 5.1(a) displays the Q-Q plot of quantiles of the data versus $G R(\theta, \beta)$. Figure 5.1 (b) display both the empirical and fitted cdf. As it can be clearly seen from Figure 5.1 (a), the quantiles of the data fall approximately on the straight line. In Figure 5.1 (b), the fitted cdf closely follows to empirical cdf.

If the GP with the GR is applied to this data, the parameter estimates obtained by using the employed estimators in the paper and the corresponding MSE values are presented in Table 5.1

From Table 5.1, it is seen that the GP outperform the RP for this data set. Besides, the GP with ML estimators have the lowest MSE value relative to other models. We present the Figure 5.2 to show the relative performances of the four GPs with the ML, the MM, the MLM and the MLS estimators and the RP. Figure 5.2 display the plots of $S_{k}, S_{k}=X_{1}+X_{2}+\ldots+X_{k}, k=1,2, \ldots, n$ and its estimates $\hat{S}_{k}, \hat{S}_{k}=\sum_{j=1}^{k} \hat{X}_{k}$, against the $k, k=1,2, \ldots, n$, where $\hat{X}_{k}$ can obtained by using (5.1).

According to Figure 5.2, it can be concluded that GPs follow true values more accurately than RP. 


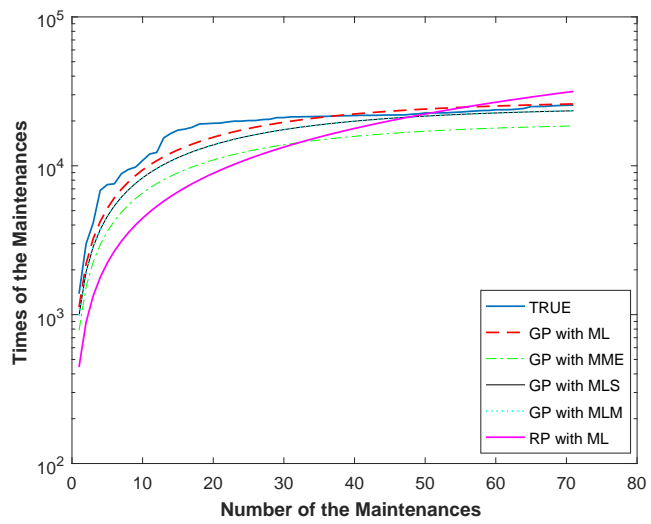

FIG. 5.2: The plots of the observed and estimated maintenance times for the No. 3 data set

Table 5.2: Estimates and evaluated $\mathrm{MSE}^{*}$ values of the different GP models for the No. 3 data

\begin{tabular}{|c|c|c|c|c|c|c|c|c|c|c|}
\hline & \multicolumn{10}{|c|}{ Model } \\
\hline & G. Rayleigh & & Gamma & & Log-Normal & & Weibull & & Inv. Gaussian & \\
\hline $\mathrm{MSE}^{*} / 10^{5}$ & 1.93257 & & 2.15623 & & 2.46508 & & 2.11300 & & 1.93442 & \\
\hline \multirow[t]{3}{*}{ Parameter Est. } & $\hat{a}$ & 1.04272 & $\hat{a}$ & 1.03547 & $\hat{a}$ & 1.04165 & $\hat{a}$ & 1.03659 & $\hat{a}$ & 1.04274 \\
\hline & $\hat{\alpha}$ & 0.12795 & $\hat{k}_{G}$ & 0.66991 & $\hat{\mu}_{L N}$ & 6.06255 & $\hat{\theta}_{W}$ & 777.7413 & $\hat{\mu}_{I G}$ & 1118.4 \\
\hline & $\hat{\lambda}$ & 0.0002 & $\hat{\theta}_{G}$ & 1290.572 & $\hat{\sigma}_{L N}$ & 1.68506 & $\hat{\lambda}_{W}$ & 0.7730 & $\hat{\sigma}_{I G}$ & 1781.1 \\
\hline
\end{tabular}



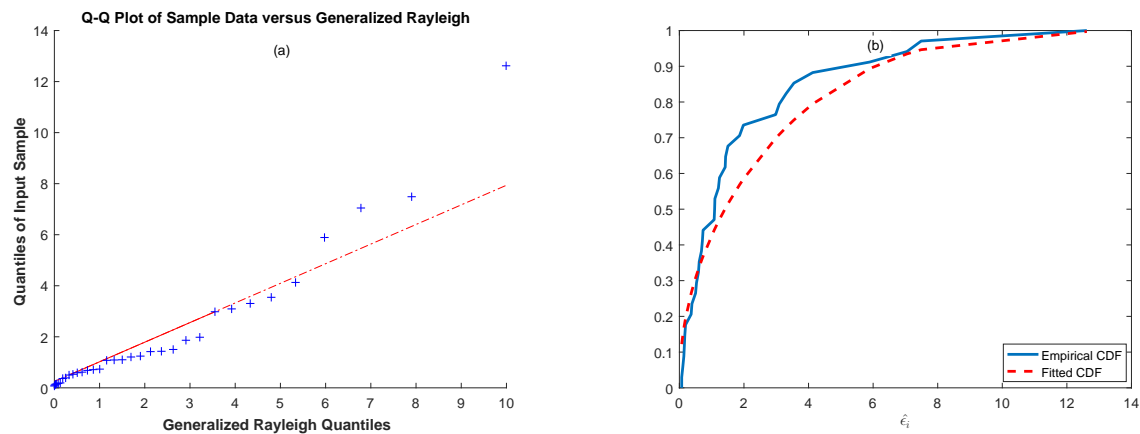

FIG. 5.3: QQ plot for the exponential errors (a), emprical and fitted cdf for the exponential errors (b)

Table 5.3: Estimation of parameters for the software data

\begin{tabular}{llcccc}
\hline Process & Method & $\hat{a}$ & $\hat{\alpha}$ & $\hat{\lambda}$ & MSE $/ 10^{3}$ \\
\hline GP & ML & 0.9094 & 0.3108 & 0.1319 & 1.8027 \\
\hline & MLS & & 0.3032 & 0.1023 & 2.1646 \\
& MM & \multirow{2}{*}{0.9370} & 0.1352 & 0.0493 & 2.0867 \\
& MLM & & 0.1293 & 0.0483 & 2.0965 \\
\hline RP & ML & 1.0000 & 0.1845 & 0.0087 & 2.6559 \\
\hline
\end{tabular}

\section{Software data:}

Software data set includes 34 observations. These data represent the time between successive failures of a piece of software developed as part of a large data system [11]. Braun et al. [9] showed that this data set consistent by a GP with the ratio parameter $a<1$. Thus we can apply a GP with the GR distribution to this data. First, we investigate whether the underlying distribution of the data is consistent with a GR distribution, as in the No. 3 data. When the regression given by (5.2) is applied to this data, estimates of the parameters for the exponential errors are $\hat{\theta}_{M L}=0.2381$ and $\hat{\beta}_{M L}=0.1677$. For this data, K-S test is 0.1615 and corresponding p-value is 0.3040 . Thus, we can say that the software data set consistent with a GR distribution. In addition, we present the Q-Q plot and the fitted and empirical cdf of the exponential errors by the Figure 5.3 to support the result of K-S test.

When a GP with the GR distribution is applied to software data set, estimates of the parameters $a, \alpha$ and $\lambda$ and the corresponding MSE values are given in Table 5.3

Acording to Table 5.3, GP outperform the RP since it has lower MSE. Furthermore, GP with the ML estimates has the best performance among all GPs. Furthermore, relative performances of the GPs with the all estimators and RP can be seen from Figure 5.4. Figure 5.4 include the plots of the $S_{k}$ and $\hat{S}_{k}$ 's against the 


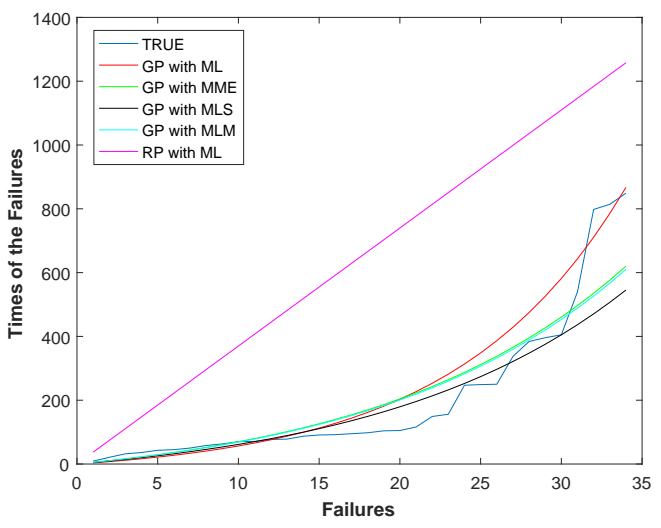

FIG. 5.4: The plots of the observed and estimated failure times for the Software data

Table 5.4: Estimates and evaluated MSE* values of the different GP models for the Software data

\begin{tabular}{|c|c|c|c|c|c|c|c|c|c|c|}
\hline & \multicolumn{10}{|c|}{ Model } \\
\hline & G. Rayleigh & & Gamma & & Log-Normal & & Weibull & & Inv. Gaussian & \\
\hline $\mathrm{MSE}^{*} / 10^{3}$ & 1.8027 & & 1.8763 & & 1.9887 & & 1.8890 & & 2.1314 & \\
\hline Parameter Est. & $\hat{a}$ & 0.9094 & $\hat{a}$ & 0.9172 & $\hat{a}$ & 0.9370 & $\hat{a}$ & 0.9186 & $\hat{a}$ & 0.9504 \\
\hline & $\hat{\alpha}$ & 0.3108 & $\hat{k}_{G}$ & 0.8533 & $\hat{\mu}_{L N}$ & 1.0017 & $\hat{\theta}_{W}$ & 3.6726 & $\hat{\mu}_{I G}$ & 7.5144 \\
\hline & $\hat{\lambda}$ & 0.1319 & $\hat{\theta}_{G}$ & 4.4649 & $\hat{\sigma}_{L N}$ & 1.2742 & $\hat{\lambda}_{W}$ & 0.8856 & $\hat{\sigma}_{I G}$ & 14.4192 \\
\hline
\end{tabular}

$k, k=1,2, \ldots, n$, where $S_{k}$ and $\hat{S}_{k}$ are defined as in the previous example.

As in the previous example, we can easily seen from Figure 5.4 that four GPs follow true values more accurately than RP.

\section{Conclusion}

The GP with the GR distribution considered by this article has many potential uses for modeling of successive arrival times observed from many fields. The process is very suitable for modeling applications of arrival times with the monotonic ascending or descending behavior as highlighted in the paper. The monotonic behavior of the GP is controlled by a positive-valued ratio parameter $a$, which is an essential feature of this process. In the paper, for the different values of the parameter $a$, the behavior of the process has been clearly illustrated in Figure 1.1. In addition to the ratio parameter $a$, the parameters of the distribution of the first arrival time are other key parameters that regulate the behavior of the process. In order to achieve an optimal modeling performance from the GP, the solution of the estimation problem of these parameters is crucial. The estimation problem for $a$, $\alpha$ and $\lambda$ parameters of GP with the GR distribution is solved by employing the 
ML methodology in the paper. The results of numerical studies which compare the efficiency of the ML estimators and modified estimators considered in this paper are presented in the tables. Tabulated results display that the ML estimators produce more efficient estimations in all cases with respect to bias and MSE criterion.

In order to demonstrate the phases of data modeling by a GP with the GR distribution and comparing its modeling performance against the RP, in the paper, two examples are carried out on real-world datasets called the No.3 and Software. In both examples, the GP with the GR distribution outperforms the RP with smaller MSE values. Furthermore, by the analysis of the results in the paper, it can be concluded that fitting by a GP with the GR distribution to both data sets is better than fitting by a GP with the possible alternatives of the GR distribution such as Gamma, Log-Normal, inverse Gaussian and Weibull.

\section{Acknowledgments}

The author thanks the anonymous referees for their comments and suggestions for improving the first version of this paper.

\section{REF EREN C E S}

1. M. Abramowitz and I. A. Irene: Handbook of mathematical functions: with formulas, graphs, and mathematical tables. Courier Corporation, 1964

2. H. Ascher and H. Feingold: Repairable Systems Reliability. Marcel Dekker, New York, 1984

3. H. AydoĞdu and B. ŞEnoĞLU and M. KARA: Parameter estimation in geometric process with Weibull distribution. Applied Mathematics and Computation. 217(6) (2010), $2657-2665$.

4. C. BIÇER: Statistical Inference for Geometric Process with the Power Lindley Distribution. Entropy, 20(10), 2018, 723.

5. C. BIÇER: Statistical inference for geometric process with the Two-parameter Rayleigh Distribution. The Most Recent Studies in Science and Art, 1, (2018), 576-583.

6. H. D. BIÇER: Statistical inference for geometric process with the Two-Parameter Lindley Distribution. Communications in Statistics-Simulation and Computation, (2019), $1-22$.

7. C. BiçER and H. D. BiçER: Statistical Inference for Geometric Process with the Lindley Distribution. Researches on Science and Art in 21st Century Turkey, 2, (2017), 2821-2829.

8. C. BiçER, and H. D. Biçer and M. KArA and H. AydoĞdu, Halil: Statistical inference for geometric process with the Rayleigh distribution. Communications Faculty of Sciences University of Ankara Series A1 Mathematics and Statistics, 68(1), 2019, 149-160.

9. W. J. Braun, and W. Li and Y. Q. ZhaO: Properties of the geometric and related processes. Naval Research Logistics. 52(7), (2005), 607-616. 
10. W. J. Braun, and W. Li and Y. Q. ZhaO: Some theoretical properties of the geometric and $\alpha$-series processes.Communications in Statistics Theory and Methods. 37(9), (2008), 1483-1496.

11. M. J. Crowder and A. C. Kimber and R. L. Smith and T. J. Sweeting: Statistical concepts in reliability. Chapman and Hall, London, 1991.

12. J. S. K. ChAn and, Y. LAM and D. Y. P. Leung: Statistical inference for geometric processes with gamma distributions. Computational statistics \& data analysis. 47(3), (2004), 565-581.

13. M. KARA and H. AYdoĞDU and Ö. TürKşEn: Statistical inference for geometric process with the inverse Gaussian distribution. Journal of Statistical Computation and Simulation. 85(16), (2015), 3206-3215.

14. D. Kundu and M. Z. RaqAB: Generalized Rayleigh distribution: different methods of estimations. Computational statistics \& data analysis. 49(1), (2005), 187-200.

15. Y. LAM: A note on the optimal replacement problem. Advances in Applied Probability. 20(2), (1988), 479-482.

16. Y. LAM: Nonparametric inference for geometric processes. Communications in statistics-theory and methods. 21(7), 1992, 2083-2105.

17. Y. LAM: The geometric process and its applications. World Scientific, 2007.

18. Y. LAM and S. K. CHAN: Statistical inference for geometric processes with lognormal distribution. Computational statistics \& data analysis. 27(1), (1998), 99-112.

19. Y. LAm and Y. Zheng and Y. Zhang: Some limit theorems in geometric processes. Acta Mathematicae Applicatae Sinica, English Series. 19(3), (2003), 405-416.

20. Y. LAm and L. ZhU and J. S. K. ChAN and Q. Liv: Analysis of data from a series of events by a geometric process model. Acta Mathematicae Applicatae Sinica, English Series. 20(2), (2004), 263-282.

21. M. Z. Raqab and D. Kundu: Burr type X distribution: revisited. Journal of probability and statistical sciences. 4(2), (2006), 179-193.

22. J.G. SURLES and W. J. PADGETT: Inference for reliability and stress-strength for a scaled Burr type X distribution. Lifetime Data Analysis, 7(2), (2001), 187-200.

Cenker Biçer

Faculty of Arts and Sciences

Department of Statistics

The University of Kırıkkale

71450 Kırıkkale, Turkey

cbicer@kku.edu.tr

Hayrinisa Demirci Biçer

Faculty of Arts and Sciences

Department of Statistics

The University of Kırıkkale

71450 Kirıkkale, Turkey

hdbicer@kku.edu.tr 
Statistical Inference for Geometric Process with Generalized Rayleigh Distribution 1125

\author{
Mahmut Kara \\ Faculty of Economics and Administrative Sciences \\ Department of Econometrics \\ The University of Yüzüncü Yıl \\ Van, Turkey \\ mkara2581@gmail.com \\ Asuman Yilmaz \\ Faculty of Economics and Administrative Sciences \\ Department of Econometrics \\ The University of Yüzüncü Yıl \\ Van, Turkey \\ asumanduva@gmail.com
}

